12 Lönnroth K, Migliori GB, Abubaker I, et al. Towards tuberculosis elimination: an action framework for low-incidence countries. Eur Respir J 2015; 45: 928-952.

13 Aouadi S, Mjid M, Maalej S, et al. Miliary tuberculosis: diagnosis difficulties and prognosis factors. Eur Respir J 2011; 38: Suppl 55, P2579.

\title{
First experience of effectiveness and safety of bedaquiline for 18 months within an optimised regimen for XDR-TB
}

\author{
To the Editor:
}

Extensively drug-resistant (XDR) tuberculosis (TB) is a type of multidrug-resistant (MDR) TB that is resistant to isoniazid, rifampicin, fluoroquinolones and at least one injectable second-line drug. There are insufficient antibiotics for effective combination therapy and mortality exceeds $70 \%$ [1]. Following successful phase IIb trials [2] in 2013, the novel mycobacterial ATP-synthase inhibitor bedaquiline was approved in Europe and the USA for the first 24 weeks of MDR/XDR-TB treatment alongside a World Health Organization (WHO)-approved optimised background regimen. Phase III trials are ongoing but cohort data describe good early bacteriological outcomes in France [3, 4], Italy [5], the UK [6], the USA [7], India [8] and South Africa [9].

However, WHO guidelines presently advocate a total MDR/XDR-TB treatment duration of $\geqslant 18$ months. In the presence of extensive resistance, antibiotic regimens may be sparse when bedaquiline is stopped. The US Centers for Disease Control and Prevention (CDC) recommend continuing bedaquiline beyond 24 weeks if effective treatment "cannot otherwise be provided", whilst acknowledging the lack of supporting tolerability data [10]. Although no fatalities in pre-licensing studies were attributed to the study drug, there were more deaths on bedaquiline than placebo (10 versus two) [2]. The plasma half-life of bedaquiline is $4-5$ months and corrected QT interval (QTc) prolongation occurs, raising concerns about incremental toxicity and highlighting the need for expedient reporting of clinical experience. Here, we describe the first published case of safe bedaquiline treatment extension to 18 months in a patient with pulmonary XDR-TB.

In January 2014, a 20-year-old, HIV-negative Romanian woman was referred to our unit with a first presentation of smear-positive pulmonary TB. Computed tomography (CT) scans showed multifocal disease of both lungs. She had no comorbidities and was initiated on first-line antituberculous chemotherapy (rifampicin, isoniazid, pyrazinamide and ethambutol) but after 2 days, the Genotype MDRTBplus 2.0 assay (Hain Diagnostics, Cookville, TN, USA), performed on the patient's sputum sample, revealed $r p o B$ and kat $G$ mutations consistent with rifampicin and isoniazid resistance. Therefore, she was switched to a WHO-approved MDR-TB regimen (figure 1). During February, full phenotypic drug susceptibility testing and whole-genome sequencing revealed extensive resistance to all first-line drugs, all quinolones, all injectables and prothionamide. Susceptibilities to linezolid, para-aminosalicylic acid (PAS) and azithromycin were confirmed. Phenotypic sensitivity testing to meropenem/imipenem and clofazimine were unavailable, but whole-genome sequencing was performed and identified $k a t G$ mutations conferring high-level isoniazid resistance, precluding the use of high-dose isoniazid. At week 8 of treatment, she was established on a six-drug regimen of bedaquiline, linezolid, pyrazinamide, cycloserine, azithromycin and PAS. Bedaquiline was obtained through a compassionate use programme and introduced at $400 \mathrm{mg}$ once daily for 2 weeks, followed by $200 \mathrm{mg}$ three times weekly. Figure 1 illustrates progression of her regimen as resistance information became available and throughout the remainder of therapy. Her sputum converted to smear and culture negative 24 days after initiation of the bedaquiline based regimen, and 81 days after starting first-line treatment.

She successfully completed her initial supply of bedaquiline. Between week 32 and 34, she developed painful peripheral neuropathy, confirmed on nerve conduction studies, which prompted discontinuation of linezolid and cycloserine. After discussion with the British Thoracic Society MDR-TB advisory panel and international experts, her remaining regimen was deemed inadequate and her bilateral pulmonary disease 

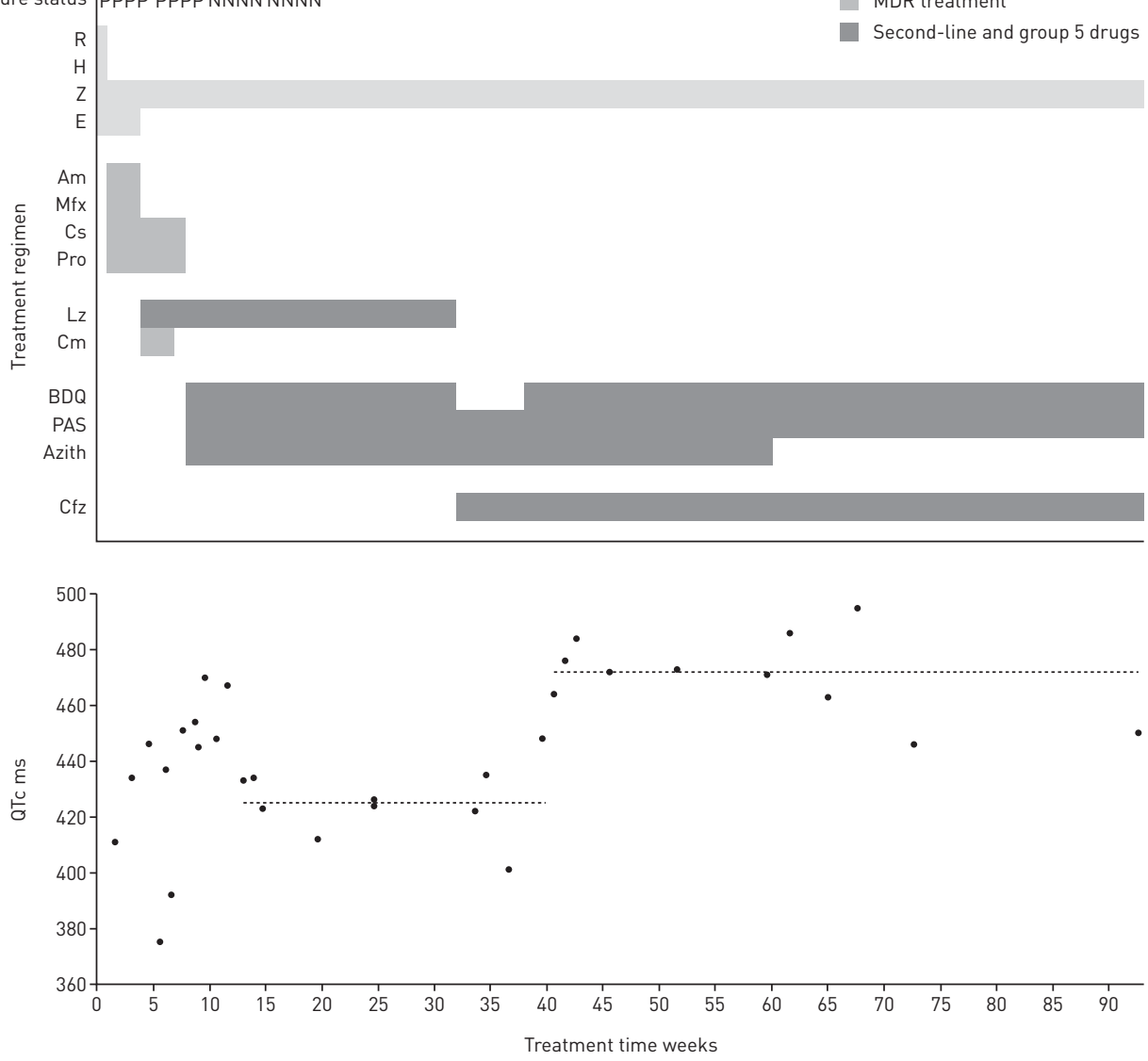

FIGURE 1 a) Drug regimen, sputum smear and culture status, and b) corrected QT interval (QTc) of the patient by week. Horizontal dashed lines represent median QTc between 12 and 39 weeks (425 ms) and 40-93 weeks (472 ms). $\mathrm{P}$ : positive sputum smear/culture; $\mathrm{N}$ : negative smear/culture; $\mathrm{R}$ : rifampicin; $\mathrm{H}$ : isoniazid; $\mathrm{Z}$ : pyrazinamide; E: ethambutol; Am: amikacin; Mfx: moxifloxacin; Cs: cycloserine; Pro: prothionamide, Lz: linezolid; Cm: capreomycin; BDQ: bedaquiline; PAS: para-aminosalicylic acid; Azith: azithromycin; Cfz: clofazimine; MDR: multidrug-resistant.

excluded surgical management. Therefore, clofazimine was introduced at week 32 and bedaquiline was restarted, at a dose of $200 \mathrm{mg}$ three times weekly, at week 38.

ECGs were reviewed by a consultant cardiologist regularly during bedaquiline administration. Figure 1 shows changes to the QTc interval over time. Initially, whilst the patient was admitted to hospital, ECGs were performed weekly, then less frequently (weekly, biweekly or monthly as directed by the cardiologist) once she was discharged; however, there were occasional periods where, due to financial or transportation problems, the patient failed to attend an ECG appointment, which is reflected in occasional periods of several weeks between ECGs in figure 1. From a pre-treatment baseline of $411 \mathrm{~ms}$, the QTc increased to $465 \mathrm{~ms}$ at week 10 before settling to a median of $425 \mathrm{~ms}$ between weeks 12 and 40 . There was a steep rise in QTc after week 40 (during bedaquiline, clofazimine and azithromycin co-administration). Azithromycin was stopped at week 62, QTc peaked at $495 \mathrm{~ms}$ in week 68 and the median QTc from week 40 until the end of therapy was $472 \mathrm{~ms}$. Serum potassium, magnesium and corrected calcium concentrations were always within normal limits, and care was taken to avoid QTc prolonging agents other than those required for TB therapy. T-wave inversion in the ECG anterior leads was also noted, though cardiac troponins were normal and the patient reported no cardiac symptoms. No cardiac arrhythmias or other adverse clinical events were reported.

Bedaquiline treatment was continued for a total of 72 weeks (18 months). With no recurrence of sputum smear or culture positivity, gradual weight gain and resolution of active inflammatory changes on serial CT scans, the patient is now regarded as cured. Post-treatment follow-up will continue to ensure there is no relapse.

This case describes that bedaquiline administration may be safely extended beyond 6 months, which is an important consideration when balancing the terrible prognosis of XDR-TB against the uncertain efficacy and safety of new antibiotics. The recognised global burden of drug-resistant TB increased from 250000 
incident cases in 2009 to 480000 cases in 2014 [11]. In Eastern Europe, over 20\% of new TB patients present with MDR or XDR disease [11] and our patient demonstrates the increasingly frequent therapeutic challenges posed by this problem.

Antibiotic options in XDR-TB are always limited to drugs of uncertain efficacy. Of the available agents, the impressive bactericidal activity of bedaquiline in phase II clinical studies [2] offers considerable promise. However, current experience and licencing of bedaquiline is limited to administration for 24 weeks of a total of $18-24$ months treatment duration [12, 13]. This will inevitably generate case management dilemmas when XDR-TB patients are left with sparse regimens for most of their therapy. To minimise the risk of eventual failure or relapse, it seems desirable to continue the most potent agents for longer and CDC guidelines allow extension of bedaquiline treatment on a case-by-case basis [10]. To our knowledge, our case is the first published description of that approach.

A major concern with prolonged bedaquiline use is the risk of toxicity. QTc interval prolongation is regarded as the most significant adverse effect [13] and we observed this, particularly when bedaquiline was administered with other known QTc prolonging agents (clofazimine and azithromycin). Nevertheless, given the high likelihood of mortality from XDR-TB treatment failure, we sought specialist cardiology input for treatment monitoring and persisted with bedaquiline. The therapeutic response was favourable and there were no clinical side-effects. It is noteworthy that our patient was young and previously well; greater caution may be required in older patients with cardiac comorbidities.

Trials are ongoing to establish whether MDR-TB therapy can be shortened to 9 months [14] and some of these studies include bedaquiline-based regimens [15]. However, it will be several years until results are available. In the meantime, clinical use of novel antituberculous drugs and combination regimens will be informed by incremental experience from case reports and case series. In this scenario, our experience that bedaquiline can be safe and effective when continued for up to 18 months in the treatment of pulmonary $\mathrm{XDR}-\mathrm{TB}$ is important and relevant for other clinicians managing drug-resistant TB.

0 @ERSpublications

The first successful use of bedaquiline for 18 months for XDR-TB as part of an optimised background regimen http://ow.ly/XE34x

Joseph M. Lewis ${ }^{1,2}$, Paul Hine ${ }^{1}$, Jenny Walker ${ }^{3}$, Saye H. Khoo $^{4}$, Miriam Taegtmeyer ${ }^{5}$, S. Bertel Squire ${ }^{5}$ and Derek J. Sloan ${ }^{5,6}$ ${ }^{1}$ Tropical and Infectious Diseases Unit, Royal Liverpool University Hospital, Liverpool, UK. ${ }^{2}$ Wellcome Trust Liverpool Glasgow Centre for Global Health Research, University of Liverpool, Liverpool, UK. ${ }^{3}$ Liverpool Community Health TB Services, Liverpool, UK. ${ }^{4}$ Dept of Pharmacology and Therapeutics, University of Liverpool, Liverpool, UK. ${ }^{5}$ Liverpool School of Tropical Medicine, Liverpool, UK. ${ }^{6}$ Liverpool Heart and Chest Hospital, Liverpool, UK.

Correspondence: Joseph M. Lewis, Tropical and Infectious Diseases Unit, Royal Liverpool University Hospital, Liverpool, L7 8XP, UK. E-mail: joseph.lewis@liverpool.ac.uk

Received: Nov 262015 | Accepted after revision: Jan 19 2016 | First published online: Feb 252016

Support statement: This work received no specific funding but Joseph M. Lewis is supported by the Wellcome Trust as a Wellcome Trust clinical PhD fellow (grant number 109105/Z/15/Z). Funding information for this article has been deposited with FundRef.

Conflict of interest: Disclosures can be found alongside the online version of this article at erj.ersjournals.com

\section{References}

1 Pietersen E, Ignatius E, Streicher EM, et al. Long-term outcomes of patients with extensively drug-resistant tuberculosis in South Africa: a cohort study. Lancet 2014; 383: 1230-1239.

2 Diacon AH, Pym A, Grobusch MP, et al. Multidrug-resistant tuberculosis and culture conversion with bedaquiline. N Engl J Med 2014; 371: 723-732.

3 Guglielmetti L, Le Dû D, Jachym $\mathrm{M}$, et al. Compassionate use of bedaquiline for the treatment of multidrug-resistant and extensively drug-resistant tuberculosis: interim analysis of a French cohort. Clin Infect Dis 2015; 60: 188-194.

4 Catho G, Couraud S, Grard S, et al. Management of emerging multidrug-resistant tuberculosis in a low-prevalence setting. Clin Microbiol Infect 2015; 21: 472.e7-e472.e10.

5 Tiberi S, De Lorenzo S, Centis R, et al. Bedaquiline in MDR/XDR-TB cases: first experience on compassionate use. Eur Respir J 2014; 43: 289-292.

6 van Halsema C, Humphreys S, Bonington A. Extensively drug-resistant tuberculosis: early access to bedaquiline for a UK patient. Eur Respir J 2014; 43: 292-294.

7 Danckers $\mathrm{M}$, Lesko MB, Adamson $\mathrm{R}$, et al. Compassionate use of bedaquiline in the treatment of pulmonary XDR-TB. Int J Tuberc Lung Dis 2014; 18: 1522-1523.

8 Udwadia ZF, Amale RA, Mullerpattan JB. Initial experience of bedaquiline use in a series of drug-resistant tuberculosis patients from India. Int J Tuberc Lung Dis 2014; 18: 1315-1318.

9 Ndjeka N, Conradie F, Schnippel K, et al. Treatment of drug-resistant tuberculosis with bedaquiline in a high HIV prevalence setting: an interim cohort analysis. Int J Tuberc Lung Dis 2015; 19: 979-985. 
10 Mase S, Chorba T, Lobue $\mathrm{P}$, et al. Provisional CDC guidelines for the use and safety monitoring of bedaquiline fumarate (Sirturo) for the treatment of multidrug-resistant tuberculosis. MMWR 2013; 62: 1-12.

11 World Health Organization. Companion Handbook to the WHO Guidelines for the Programmatic Management of Drug-Resistant Tuberculosis. Geneva, WHO, 2014.

12 Falzon D, Jaramillo E, Schünemann HJ, et al. WHO guidelines for the programmatic management of drug-resistant tuberculosis: 2011 update. Eur Respir J 2011; 38: 516-528.

13 Pontali E, Sotgiu G, D’Ambrosio L, et al. Bedaquiline and multidrug-resistant tuberculosis: a systematic and critical analysis of the evidence. Eur Respir J 2016; 47: 394-402.

14 Aung KJM, Van Deun A, Declercq E, et al. Successful "9-month Bangladesh regimen" for multidrug-resistant tuberculosis among over 500 consecutive patients. Int J Tuberc Lung Dis 2014; 18: 1180-1187.

15 IUALTD, Inc. The Evaluation of a Standard Treatment Regimen of Anti-tuberculosis Drugs for Patients with MDR-TB (STREAM). https://clinicaltrials.gov/ct2/show/record/NCT02409290. Date last accessed: July 16, 2015. Date last updated: April 3, 2015.

\section{An overview on tuberculosis-specific hospitals in China in 2009: results of a national survey}

To the Editor:

As of 2014, tuberculosis (TB) was the deadliest infectious disease worldwide and China has the third-highest TB burden [1]. In that year alone there were 826155 new cases in China [1]. In addition, China has been experiencing a serious epidemic of drug-resistant TB [2, 3].

The availability of adequately equipped microbiology laboratories is an essential element of effective TB control $[4,5]$. The Chinese National Center for TB control (NCTB), which was organised through the public health system throughout the 1990s and early 2000s, invested in this area. As many as $3490 \mathrm{~TB}$ dispensaries were established [6]. In 2005, \$1.3 billion was invested to rebuild 2448 dispensaries and scale up new TB tests such as liquid culture $[7,8]$.

Since early 2000, the NCTB programme has been moving TB care towards a hospital-based management model which mandates tuberculosis-specific hospitals to diagnose and treat TB patients [8]. These hospital are public hospitals or health facilities and are designated by their local authority to clinically manage TB patient. Variants include TB hospitals, chest hospitals and infectious disease hospitals, etc. [8]. At the moment, there is little knowledge regarding the testing capacity of laboratories within these hospitals. Additionally, there is also little data about the prevalence of multi drug-resistant TB (MDR-TB) in tuberculosis-specific hospitals. The latest national MDR-TB survey in China was conducted only in TB dispensaries, i.e. within the public health system. In this study, we aim to address the aforementioned issues by conducting a national survey in microbiology laboratories in tuberculosis-specific hospitals in 2009.

The survey was conducted using a standardised questionnaire designed by the National Tuberculosis Clinical Center. We collected cross-sectional information from all 203 tuberculosis-specific hospitals in China in existence in 2009 with at least 30 beds in TB wards. The study was conducted in 31 provinces, municipalities, and autonomous regions, with questionnaires completed by directors of $\mathrm{TB} /$ respiratory department, physicians, and laboratory technicians. A training course for data collection officials was held at the National Tuberculosis Clinical Center to ensure data integrity.

The questionnaire gathered information on the usage of four TB tests, and the number of drug-resistant TB cases diagnosed. The four tests include sputum smear microscopy, solid and liquid culture, and phenotypic drug susceptibility test (DST), which are recommended by the 2008 National Guidelines [9]. Because other tests, such as nucleic acid amplification test, were not approved in China for detection of TB between 1999 and 2009, data on these tests had not been regularly collected by TB hospitals and could not be included. Testing capacity data for the 203 tuberculosis-specific hospitals was retrieved for the year 2004 and 1999. Overall test volume per hospital and average number of tests performed per hospital were recorded on an annual basis. Since these hospitals had not participated in external quality assessment until 2010, we had no 